Relations industrielles

Industrial Relations

\title{
Hogue, Jean-Pierre et Pierre Brulé, La puissance du stress : une valeur ajoutée
}

\section{Alain Larocque}

Volume 55, numéro 2, 2000

URI : https://id.erudit.org/iderudit/051322ar

DOI : https://doi.org/10.7202/051322ar

Aller au sommaire du numéro

Éditeur(s)

Département des relations industrielles de l'Université Laval

ISSN

0034-379X (imprimé)

1703-8138 (numérique)

Découvrir la revue

Citer ce compte rendu

Larocque, A. (2000). Compte rendu de [Hogue, Jean-Pierre et Pierre Brulé, $L a$ puissance du stress : une valeur ajoutée]. Relations industrielles / Industrial Relations, 55(2), 375-376. https://doi.org/10.7202/051322ar

Tous droits réservés @ C Département des relations industrielles de l'Université Laval, 2000
Ce document est protégé par la loi sur le droit d'auteur. L’utilisation des services d'Érudit (y compris la reproduction) est assujettie à sa politique d'utilisation que vous pouvez consulter en ligne.

https://apropos.erudit.org/fr/usagers/politique-dutilisation/ 
le poste (Lawler, Journal of Organizational Behavior, 1993). Comment s'assurer que la mise en place de la gestion par les compétences ne viendra pas raviver la discrimination systémique en emploi comme l'ont montré Moss et Tilly au sujet des « soft skills » (Work and Occupations, 1996, $\mathrm{n}^{\circ} 3$ ) ?

Ces commentaires critiques, qui s'adressent en fait à la plupart des promoteurs de la gestion par les compé- tences, ne doivent pas faire oublier les qualités de cet ouvrage. Il demeure un de ceux qui vont le plus loin dans l'explication de certains enjeux de la gestion par les compétences et dans la compréhension de la notion de compétence, entre autres son analyse de la relation souvent antagonique entre la performance et la compétence (p. 48-51).

GUY BELLEMARE Université du Québec à Hull

\section{La puissance du stress : une valeur ajoutée}

par Jean-Pierre Hogue et Pierre Brulé, Cap-Rouge et Montréal : Presses Inter Universitaires et Presses HEC, 1997, 167 p., ISBN 2-8944-035-2.

Le stress demeure un phénomène complexe qui touche aussi bien les individus, les organisations que les sociétés. Dans nos sociétés industrielles, la recherche de moyens efficaces pour le contrôler et en tirer avantages a inspiré de très nombreuses publications qui mettent de l'avant les vertus positives du stress comme facteur de croissance personnelle.

La contribution des auteurs Hogue et Brulé se situe dans cette perspective. Le stress est ainsi une énergie qui résulte de l'interaction d'un individu avec son environnement. Tout est énergie, tout est stress, affirment-ils en introduction. Le fil conducteur de l'ouvrage repose sur l'affirmation que le stress est au cœur même de la vie : sans stress, il n'y a pas de vie. Ils se situent dans la même perspective qu'Hans Selye, «le père du stress » qui, dans l'un de ses derniers écrits (Stress sans détresse, 1974), considérait le stress comme un moyen sans lequel il n'est pas possible de s'adapter à son environnement.

Le volume se divise en six chapitres. Les quatre premiers présentent le stress comme une affaire personnelle, une façon d'être et de percevoir son environnement : toute adaptation demeure relative à la perception subjective que l'individu a de son environnement. Selon qu'un environnement est perçu comme peu dangereux, dangereux ou très dangereux, il mobilisera des énergies, des ressources variables à son contrôle, énergies non disponibles pour d'autres fins. Le tout demeure lié à la représentation que chacun se fait du danger, représentation à l'origine de la mobilisation des énergies.

Ainsi, au chapitre I, les auteurs présentent le stress au centre du rythme des changements et des transformations qui bouleversent, déséquilibrent et forcent les gens à l'adaptation constante à leur environnement. Face à ces changements, ils affirment, au chapitre II, que chacun est amené à développer une façon d'être, à partir des énergies générées par le stress, pour vivre et continuer de négocier avec l'environnement.

Le chapitre III présente une comparaison du comportement humain avec le comportement du gestionnaire qui doit transiger dans un environnement en constant changement. Se référant à Mintzberg et à Covey, les auteurs poussent l'analogie en l'appuyant sur la nécessité de gérer l'incertitude l'intégrant à l'émotif, l'intuitif et... au gros bon sens. La démonstration convainc peu en ce qu'elle passe sous silence toutes les données de la recherche dans ce domaine, mais elle fait image. Au chapitre IV, les auteurs associent le stress au phénomène de perception résultant « d'un 
débalancement perceptuel d'un individu ». Cette vision phénoménologique du stress fait appel, selon eux, à la personnalité, la conscience et la prise en charge de soi.

Les deux autres chapitres visent à démontrer que le stress n'apporte pas qu'aspects négatifs et détresse. Bien utilisé, il peut constituer un acquis pour l'individu et devenir une énergie d'adaptation ou, selon leurs termes, une valeur rentable (une valeur ajoutée). Ainsi, le chapitre $\mathrm{V}$ présente les aspects positifs qui peuvent résulter du stress s'il est canalisé dans des activités structurées de dépassement qui permettent à la personne d'accroître son identité. Au dernier chapitre, les auteurs Hogue et Brulé visent à établir un lien entre l'individu et l'organisation. Ce lien, selon eux, résiderait dans le pouvoir intégrateur du stress comme énergie de dépassement. Bref, le lecteur est amené à considérer le stress comme un phénomène puissant et paradoxal aux multiples vertus !

Il ne faut pas chercher dans ce volume une étude systématique et rigoureuse du stress, ni y voir une préoccupation de présenter des moyens concrets pour en gérer efficacement l'occurrence. Pas plus d'ailleurs qu'il ne faut y chercher une étude sur l'impact du stress dans les organisations ou les milieux de travail. Il s'agit plutôt d'une introduction générale au phénomène du stress dans une perspective humaniste et phénoménologique pour laquelle Hogue et Brulé réfèrent fréquemment à la comparaison et l'analogie pour convaincre. Les auteurs présentent le stress sous son jour positif comme une valeur ajoutée pour l'individu qui sait l'utiliser à son profit tout en le laissant à lui-même pour trouver les moyens d'y parvenir.

La personne désireuse de s'éveiller à cette réalité très actuelle que constitue le stress ou encore celle qui participe à un séminaire de formation sur le stress trouvera sûrement profit à lire cet ouvrage facile d'accès. Il n'est pas destiné aux gens déjà informés dans le domaine qui auront tendance à le trouver général, sans rapport précis avec la recherche actuelle dans le domaine.

Alain Larocque Université Laval 